\title{
SARS-CoV-2 association with hemoglobin and iron metabolism
}

\author{
Elaine Maria Borges Mancilha1* (D), José Salvador Rodrigues de Oliveira²
}

\section{INTRODUCTION}

In March 2020, the World Health Organization (WHO) declared COVID-19 pandemic, a disease related to the new coronavirus that causes a severe acute respiratory syndrome, which was named as SARS-CoV-2. The severe form of this disease presents pulmonary involvement in the form of aggressive and extensive interstitial pneumonia, which is triggered by a sustained systemic acute inflammatory response known as "cytokine storm." This has been previously described in the literature as a complication of a group of diseases that can be categorized under the term "hyperferritinemic syndrome." Its exponents include hemophagocytic syndrome, adult Still's disease, catastrophic antiphospholipid syndrome, and septic shock ${ }^{1,2}$.

It has previously been demonstrated that SARS-CoV-2 binds to hemoglobin through ACE2, CD147, Cd26, and other receptors that are present on the surface of erythrocytes. After this association, virus attacks the betal chain of hemoglobin which leads to dysfunctional hemoglobin in addition to hemolysis, thereby reducing the oxygen supply to the body, causing tissue hypoxia, a remarkable characteristic of COVID-193,.

Esaki demonstrated that the amino acid sequence of the coronavirus spike protein is identical to hepcidin, a protein that acts as the main systemic regulator of iron metabolism. Therefore, this similarity between hepcidin and coronavirus spike protein can lead to a mimetic effect, suggesting that SARS-CoV-2 can increase serum hepcidin and then ferritin, and cause hyperferritinemic syndrome ${ }^{5}$.

Therefore, the association between dysfunctional hemoglobinopathy and SARS-CoV-2-related hyperferritinemia may affect the oxygen transport capacity of erythrocytes, thereby leading to hypoxia, while causing tissue damage due to non-transferrin bound iron (NTBI), and subsequently releasing free radicals at the inflammation sites ${ }^{6}$.

\section{REVIEW ON IRON \\ METABOLISM AND HEPCIDIN}

Iron $(\mathrm{Fe})$ metabolism is mainly regulated by the coordination between erythropoiesis and Fe stores. Two mechanisms are mainly involved in the regulation of this homeostasis: the intracellular mechanism that is dependent on the cytoplasmic Fe store, and the systemic mechanism in which hepcidin plays a crucial role.

\section{Intracellular Fe homeostasis}

Dietary $\mathrm{Fe}^{2+}$ is absorbed after binding to heme carrier protein 1 (HCP1) in the brush border membrane of the duodenal enterocytes and HCP1 imports it into the intracellular medium. $\mathrm{Fe}^{2+}$ is then released from the protoporphyrin by heme oxygenase and can be stored as ferritin or exported to the blood ${ }^{7,8}$.

Intracellular $\mathrm{Fe}^{2+}$ is exported to the plasma through ferroportin (FPT), a Fe exporting protein, and after the action of hephaestin, $\mathrm{Fe}^{2+}$ is transformed into $\mathrm{Fe}^{3+}$ that binds to transferrin (Tf) and circulates in the plasma ${ }^{7,8}$.

There is no specific mechanism to eliminate the excessive iron resulting from the cellular uptake and recycling of red blood cells. Therefore, the homeostasis of serum Fe requires a coordination between the sites of absorption, utilization, and storage; this signaling is conducted by hepcidin ${ }^{7,8}$.

\section{Systemic iron homeostasis}

Hepcidin is a peptide that acts as a negative regulator of $\mathrm{Fe}$ metabolism ${ }^{9,10}$. It has the ability to bind to FPT, located in the enterocytes and bone marrow, in addition to being present in macrophages of the liver and spleen, the organs that are also responsible for filtering senescent red blood cells and storing intracytoplasmic $\mathrm{Fe}^{11}$.

When serum hepcidin is at high levels, the hepcidin-FPT complex is formed and hepcidin blocks the release of Fe from

\footnotetext{
'Universidade Federal de São Paulo - São Paulo (SP), Brazil.

¿Universidade Federal de São Paulo, Department of Clinical and Experimental Oncology - São Paulo (SP), Brazil.

*Corresponding author: elaine_mancilha@hotmail.com

Conflicts of interest: the authors declare there are no conflicts of interest. Funding: none.

Received on June 04, 2021. Accepted on July 03, 2021.
} 
the macrophage reticuloendothelial system. As a result, Fe accumulates in the hepatocytes and macrophages. The decrease in plasma Fe levels results in a low transferrin saturation (TS) ratio and less stimulus for erythropoiesis ${ }^{9}, 10$.

Regarding the intestinal absorption of Fe, hepcidin inhibits iron uptake by the enterocytes through binding with FPT of the basolateral membrane and thus its consequent internalization ${ }^{12}$.

Serum Fe levels regulate hepcidin expression. In case of iron overload (IO), its expression is increased, while in conditions such as anemia and hypoxemia its expression is generally reduced. In an acute inflammatory state, as well as in COVID19 , IL-6 acts directly on the hepatocytes, stimulating the production of hepcidin and consequently leading to a decrease in TS and an increase in intracytoplasmic ferritin ${ }^{7,8}$.

\section{Plasma iron circulation}

Transferrin (Tf) is the protein responsible for the transport of Fe through plasma, and it promotes auto-oxidation reactions involving $\mathrm{Fe}$ and prevents the formation of oxygen free radicals (reactive oxygen species [ROS]) in the bloodstream, which will occur if $\mathrm{Fe}$ is transported in its free and toxic form.

Fe-saturated Tf binds to the transferrin receptor (TfR) present on the cell surface, which is available in two isoforms, namely, $\mathrm{TfR} 1$ and TfR $2^{13}$. The most recent studies suggest that while TfR 1 plays a crucial role in Fe endocytosis, TfR2 functions as a sensor of the available Fe pool in the body and modulates the production of hepcidin in the liver through the activation of the BMP pathway ${ }^{14}$.

Tf competes with human hemochromatosis protein (HFE) for TfR1 binding ${ }^{8,9}$. HFE protein while interacting with TfR1 detects the degree of TS and signals the hepatocytes whether there is a need for Fe absorption in the intestinal lumen, and based on this information, the production of hepcidin is stimulated. Under conditions of high TS, there is increased binding of Tf to its receptor, and subsequently, free HFE signals the nucleus to induce hepcidin synthesis.

\section{Iron storage}

Ferritin is a molecule that comprises 24 heavy chain $(21 \mathrm{kDa})$ and light chain $(19 \mathrm{kDa})$ subunits. It is synthesized by the liver and exhibits the function of being an easily accessible intracellular Fe store ${ }^{13,14}$.

The synthesis of ferritin subunits is regulated by RNA transcription in the hepatocytes, which is induced after the binding of iron regulatory proteins (IRP) to an iron responsive element (IRE). When intracellular Fe concentration is low, the binding of IRP to IRE suppresses the response for the production of ferritin. Conversely, when the intracellular Fe concentration is high, IRP is degraded, making its binding to the IRE impossible and then leading to ferritin synthesis ${ }^{13,15}$.
Serum ferritin (SF) concentration is a reliable marker of the body Fe reserves ${ }^{11}$. In situations where ferritin level is increased, as it is an acute phase protein, it is always necessary to assess the underlying existence of inflammatory diseases, infectious diseases, and neoplasms. It is necessary to assess whether there is an IO that can be determined by high TS?

In humans, the ideal TS level is $30 \%$, and thus, when it is less than $16 \%$, there is Fe deficiency, a characteristic of iron deficiency anemia. However, when it is greater than $45 \%$, there are signs of IO. When TS is $>70 \%$, a free form of iron begins to accumulate in the plasma, known as non-transferrin bound iron (NTBI) ${ }^{4,6}$, thereby causing $\mathrm{IO}$ in the parenchymal cells. Free Fe catalyzes the conversion of free radicals from oxygen. Free ROS in the cytoplasm leads to the damage of organelles, especially the DNA in the nucleus and mitochondria ${ }^{9,10}$.

\section{DYSFUNCTIONAL HEMOGLOBINOPATHY IN COVID-19}

The literature reinforces the hypothesis of the involvement of erythrocytes in COVID-19; the free heme resulting from hemolysis present in the bloodstream of a patient who is undergoing "cytokine storm," associated with hyperferritinemia, contributes to endothelial damage and to the remodeling of pulmonary vessels ${ }^{6}$. The destruction of erythrocytes leads to anemia and consequently hypoxia, thereby leading to systemic vasodilation, but with pulmonary vasoconstriction, it leads to an increase in fibrinogenesis in the pulmonary microvasculature. The increase in IL-6 in the inflammatory phase of COVID-19 increases hepcidin, which further leads to pulmonary hypertension through stimulating myocytes in the pulmonary alveoli, in addition to alveolar wall exudation secondary to local inflammation $^{15,16}$.

The formation of oxygen free radicals on hemolysis of erythrocytes and release of free Fe leads to mitochondrial damage in the activated macrophages and pulmonary endothelium, further perpetuating the inflammatory process and hyperstimulating the release of cytokines, especially IL-6. Other inducers for the production of hepcidin are obesity and high level of glycated hemoglobin, which increase the expression of cd147 in hemoglobin, and this association increases the risk of further complications ${ }^{9}$.

\section{INCREASED HEPCIDIN IN COVID-19}

The role of iron toxicity in the pathophysiology of COVID19 is related to the hepcidin mimetic effect of SARS$\mathrm{CoV}-2$, with consequent internalization of ferroportin, 
both in the gastrointestinal tract and reticuloendothelial system, thereby causing a blockage in the availability of SF, which leads to anemia and hyperferritinemia, and ultimately ferroptosis ${ }^{5,17}$.

Through mimicking the action of hepcidin, SARS-CoV-2 exaggeratedly increases the concentration of intratissue (e.g., liver, spleen, bone marrow, and muscles)ferritin, while there is a reduction in the availability of SF, and consequently, a reduction in erythrocyte production. This decrease in circulating erythrocytes perpetuates systemic hypoxemia and hinders tissue oxygenation, which is already impaired in patients with acute respiratory syndrome.

\section{OXIDATIVE STRESS AND FERROPTOSIS}

SARS-CoV-2 infection also causes mitochondrial dysfunction in bronchial epithelial cells and macrophages, and therefore, the mitochondria becomes dysfunctional for regulating intracytoplasmic Fe metabolism with an increase in ROS, and the process culminates in what may be referred to as ferroptosis ${ }^{18}$.

Excess Fe can be tolerated to a limited extent, as is the case of silent hypoxia. Ferroptosis associated with multiorgan oxidative stress can precipitate the "cytokine storm" in later stages of the disease, for instance, in critically ill patients. The laboratory examination of critically ill patients indicates low hemoglobin and high ferritin levels in non-surviving patients ${ }^{19}$.

Tissue Fe sequestration results in an increase in ferritin in the pulmonary epithelium, which is still associated with an increase in cytokines and immune cells, such as lymphocytes and monocytes, in the pulmonary capillaries. This is probably related to the physiological need to protect the lung cells from oxygen deprivation ${ }^{20}$.

\section{SYSTEMIC HYPOXEMIA AND HYPERFERRITINEMIA}

Patients with COVID-19 exhibit silent hypoxia described as hypoxemia, which is associated with normal capnia reflecting normal gaseous exchange. Since $\mathrm{CO}_{2}$ is the body dyspnea sensor, the patients present only dyspnea in the final stages of the disease when $\mathrm{CO}_{2}$ is extremely high and there is progression of hyperferritinemia affecting the integrity of the alveolar capillary membrane, along with inflammation, edema, and pulmonary cell necrosis ${ }^{16}$.

According to Sonnweber et al. ${ }^{21}$, patients with severe SARSCoV-2 exhibited persistent hyperferritinemia even after 2 months of disease, however, without persistent levels of inflammatory markers, such as C-reactive protein (CRP) and IL-6. Patients with persistent hyperferritinemia still exhibited greater pulmonary involvement on computed tomography (CT) scans and worsened performance status after a long period of illness, which indicates that hyperferritinemia in these cases is not only an inflammatory marker, but also it exerts a direct influence on the pathophysiology of the disease ${ }^{21}$.

Interaction of SARS-CoV-2 with iron metabolism and low oxygen supply may be related to ancestral phylogenetic mechanisms, which date back to environments with low oxygen and highly available Fe levels. Alternatively, the evolution of viral replication is well adapted to this type of microenvironment, where the Fenton oxidative reaction is favored ${ }^{22,23}$.

Viruses generally stimulate increased iron deposition in the host cells. Conversely, the immune system tends to control excess iron through increasing TSAT, with the stimulation to TFR 1 and TFR 2 iron saturation sensors, receptors that can be used by viruses to enter the host cells ${ }^{24}$.

Generally, laboratory findings commonly found in patients with COVID-19, such as low hemoglobin, hyperferritinemia, low serum iron, thrombocytopenia, increased RDW and DHL, suggest that the hypothesis of the dysregulation of iron metabolism associated with inefficient erythropoiesis is a possible mechanism underlying the pathophysiological changes in patients with COVID-19.

COVID-19 combines hypoxic anemia (low hemoglobin concentration) with hypoxemic hypoxia (low hemoglobin saturation). Oxygen deprivation and iron accumulation in the lungs cause pulmonary vasoconstriction and shunting despite pneumonia ${ }^{25}$. These changes can be observed in the pathognomonic clinical manifestation of SARS-CoV-2, in which the patients present severe oxygen desaturation without presenting hypercapnia and no compensatory tachypnea.

\section{CONCLUSIONS}

SARS-CoV-2 is a disease that causes silent hypoxia associated with a severe hyperinflammatory state that triggers a "cytokine storm" along with persistent hyperferritinemia and systemic hypoxemia, and severe endothelial damage to the lung parenchyma caused by the free radicals. Since hyperferritinemia is not only an inflammatory marker but also actively causes tissue damage, its mechanism of action needs to be elucidated to broaden our understanding in the future studies.

\section{AUTHORS' CONTRIBUTIONS}

EMBM: Writing - original draft, Writing - review \& editing. JSRO: Supervision, Writing - review \& editing. 


\section{REFERENCES}

1. Rosário C, Zandman-Goddard G, Meyron-Holtz EG, D’Cruz DP, Shoenfeld Y. The hyperferritinemic syndrome: macrophage activation syndrome, Still's disease, septic shock and catastrophic antiphospholipid syndrome. BMC Med. 2013;11:185. https:// doi.org/10.1186/1741-7015-11-185

2. Shoenfeld Y. Corona (COVID-19) time musings: our involvement in COVID-19 pathogenesis, diagnosis, treatment and vaccine planning. Autoimmun Rev. 2020;19(6):102538. https://doi. org/10.1016/j.autrev.2020.102538

3. Cavezzi A, Troiani E, Corrao S. COVID-19: hemoglobin, iron, and hypoxia beyond inflammation. A narrative review. Clin Pract. 2020;10(2):1271. https://doi.org/10.4081/cp.2020.1271

4. Abrahams L. Covid-19: acquired acute porphyria hypothesis. OSF Preprints. 2020. https://doi.org/10.31219/osf.io/fxz3p

5. Ehsani S. COVID-19 and iron dysregulation: distant sequence similarity between hepcidin and the novel coronavirus spike glycoprotein. Biol Direct. 2020;15(1):19. https://doi. org/10.1186/s13062-020-00275-2

6. Varga Z, Flammer AJ, Steiger P, Haberecker M, Andermatt R, Zinkernagel AS, et al. Endothelial cell infection and endotheliitis in COVID-19. Lancet. 2020;395(10234):1417-8. https://doi. org/10.1016/S0140-6736(20)30937-5

7. Grotto HZW. Metabolismo do ferro: uma revisão sobre os principais mecanismos envolvidos em sua homeostase. Rev Bras Hematol Hemoter. 2008;30(5):390-7. https://doi.org/10.1590/ S1516-84842008000500012

8. Lopes AC. Tratado de Clínica Médica. 2th Ed. São Paulo: Editora Loca LTDA; 2009. p.1921-5.

9. De Domenico I, Ward DM, Kaplan J. Hepcidin regulation: ironing out the details. J Clin Invest. 2007;117(7):1755-8. https://doi.org/10.1172/JCI32701

10. Poli M, Asperti M, Ruzzenenti P, Regoni M, Arosio P. Hepcidin antagonists for potential treatments of disorders with hepcidin excess. Front Pharmacol. 2014;5:86. https://doi.org/10.3389/ fphar.2014.00086

11. Beucher G, Grossetti E, Simonet T, Leporrier M, Dreyfus M. Iron deficiency anemia and pregnancy. Prevention and treatment. J Gynecol Obstet Biol Reprod (Paris). 2011;40(3):185-200. https://doi.org/10.1016/j.jgyn.2011.01.004

12. Gupta A, Manaktala U, Rathore AM. A randomised controlled trial to compare intravenous iron sucrose and oral iron in treatment of iron deficiency anemia in pregnancy. Indian J Hematol Blood Transfus. 2014;30(2):120-5. https://doi. org/10.1007/s12288-012-0224-1

13. Kawabata H, Fleming RE, Gui D, Moon SY, Saitoh T, O'Kelly J, et al. Expression of hepcidin is down-regulated in TfR2 mutant mice manifesting a phenotype of hereditary hemochromatosis. Blood. 2005;105(1):376-81. https://doi.org/10.1182/blood2004-04-1416
14. Zhang D, Albert DW, Kohlhepp P, D-Pham DQ, Winzerling JJ. Repression of Manduca sexta ferritin synthesis by IRP1/ IRE interaction. Insect Mol Biol. 2001;10(6):531-9. https:// doi.org/10.1046/j.0962-1075.2001.00293.x

15. Ramakrishnan L, Pedersen SL, Toe QK, West LE, Mumby S, Casbolt $\mathrm{H}$, et al. The Hepcidin/Ferroportin axis modulates proliferation of pulmonary artery smooth muscle cells. Sci Rep. 2018;8(1):12972. https://doi.org/10.1038/s41598-018-31095-0

16. Neves J, Haider T, Gassmann M, Muckenthaler MU. Iron homeostasis in the lungs-a balance between health and disease. Pharmaceuticals (Basel). 2019;12(1):5. https://doi. org/10.3390/ph12010005

17. Hirschhorn T, Stockwell BR. The development of the concept of ferroptosis. Free Radic Biol Med. 2019;133:130-43. https:// doi.org/10.1016/j.freeradbiomed.2018.09.043

18. Radzikowska U, Ding M, Tan G, Zhakparov D, Peng $Y$, Wawrzyniak P, et al. Distribution of ACE2, CD147, CD26, and other SARS-CoV-2 associated molecules in tissues and immune cells in health and in asthma, COPD, obesity, hypertension, and COVID-19 risk factors. Allergy. 2020;75(11):2829-45. https://doi.org/10.1111/all.14429

19. Wang D, Hu B, Hu C, Zhu F, Liu X, Zhang J, et al. Clinical characteristics of 138 hospitalized patients with 2019 novel Coronavirus-Infected Pneumonia in Wuhan, China. JAMA. 2020;323(11):1061-9. https://doi.org/10.1001/jama.2020.1585

20. Zhou F, Yu T, Du R, Fan G, Liu Y, Liu Z, et al. Clinical course and risk factors for mortality of adult inpatients with COVID-19 in Wuhan, China: a retrospective cohort study. Lancet. 2020;395(10229):1054-62. https://doi.org/10.1016/S01406736(20)30566-3

21. Sonnweber $T$, Boehm A, Sahanic S, Pizzini A, Aichner $M$, Sonnweber $B$, et al. Persisting alterations of iron homeostasis in COVID-19 are associated with non-resolving lung pathologies and poor patients' performance: a prospective observational cohort study. Respir Res. 2020;21(1):276. https://doi. org/10.1186/s12931-020-01546-2

22. Eren E, Tuzkaya UR. Safe distance-based vehicle routing problem: medical waste collection case study in COVID-19 pandemic. Comput Ind Eng. 2021;157:107328. https://doi. org/10.1016/j.cie.2021.107328

23. Schmidt SM. The role of iron in viral infections. Front Biosci (Landmark Ed). 2020;25:893-911. https://doi.org/10.2741/483

24. Wessling-Resnick M. Crossing the iron gate: why and how transferrin receptors mediate viral entry. Annu Rev Nutr. 2018;38:431-58. https://doi.org/10.1146/annurevnutr-082117-051749

25. Tobin MJ. Basing Respiratory Management of COVID-19 on Physiological Principles. Am J Respir Crit Care Med. 2020;201(11):1319-20. https://doi.org/10.1164/rccm.2020041076ED 